\title{
Pericarditis purulenta primaria por Streptococcus pneumoniae, a propósito de un caso clínico
}

\author{
ALEJANDRO DONOSO F. ${ }^{1}$, FRANCO DÍAZ R. ${ }^{2}$, KATALINA BERTRÁN S. ${ }^{3}$, PABLO CRUCES R. ${ }^{.}$ \\ 1. Pediatra Intensivista. Área de Cuidados Críticos. Hospital Padre Hurtado. \\ 2. Becado Medicina Intensiva Pediátrica, Facultad de Medicina Clínica Alemana Universidad del Desarrollo. \\ 3. Interna Medicina. Facultad de Medicina Clínica Alemana Universidad del Desarrollo.
}

\begin{abstract}
Primary purulent pericarditis by Streptococcus pneumoniae. Case - report and literature review

Background: Purulent pericarditis has become a rare clinical entity since the onset of antimicrobial therapy and has a poor outcome in the majority of cases. Case-report: A healthy 3 month-old patient admitted with dyspnea, pallor and anorexia, developing cardiogenic shock due to cardiac tamponade. Chest X-ray showed cardiomegaly. He required mechanical ventilation, volume resuscitation and vasoactive drugs. Echocardiogram showed a large pericardial effusion, CT scan ruled out lung and mediastinal infection. Pericardial drainage was performed and Vancomycin plus Ceftriaxone were initiated, with a positive blood culture for Penicillin-sensitive Streptococcus pneumoniae. The evolution was favourable after surgical drainage and controlling the infection. No extraperdicardial infection was found. He received 3 weeks of antibiotic therapy. Immunological studies were normal. Conclusion: Primary purulent pericarditis is uncommon, so early detection and treatment of this life-threatening condition may lead to a good outcome. (Key words: Pericarditis, purulent pericarditis, cardiac tamponade, Streptococcus pneumoniae).
\end{abstract}

Rev Chil Pediatr 2008; 79 (6): 623-628

\section{RESUMEN}

Hoy en día la pericarditis purulenta (PP) es una patología poco frecuente, pero de pronóstico grave. Comunicamos el caso clínico de un paciente de 3 meses, sano previamente. Consultó por palidez, rechazo alimentario y dificultad respiratoria de pocas horas de evolución. La radiografía de tórax demostró cardiomegalia. Evolucionó hacia shock cardiogénico por taponamiento cardíaco. Recibió inicialmente expansores de volumen y drogas vasoactivas. Antibioterapia con vancomicina y ceftriaxona. Ecocardiograma objetivó derrame pericárdico extenso, complementado con TAC que descartó foco infeccioso endotoráxico. Se realizó pericardiocentesis y luego ventana pericárdica. Se aisló en hemocultivo

Trabajo recibido el 14 de abril de 2008, devuelto para corregir el 01 de septiembre de 2008, segunda versión el 16 de septiembre de 2008, aceptado para publicación el 20 de octubre de 2008.

Correspondencia a:

Franco Díaz Rubio

E-mail: fdiazr@gmail.com 
Streptococcus pneumoniae, sensible a penicilina. Luego de drenaje quirúrgico y control de infección presentó evolución favorable. No se encontró sitio infeccioso extrapericárdico. Completó tres semanas de tratamiento antibiótico. Estudio inmunológico fue normal. La PP es observada raramente en individuos sanos. La presentación en este caso fue de horas, por un agente inhabitual y de extrema gravedad. Un diagnóstico precoz, en conjunto con un tratamiento médico-quirúrgico es fundamental, como la mejor forma de evitar secuelas.

(Palabras clave: Pericarditis, pericarditis purulenta, taponamiento cardíaco, Streptococcus pneumoniae). Rev Chil Pediatr 2008; 79 (6): 623-628

\section{Introducción}

La pericarditis purulenta (PP) es una enfermedad grave, clásicamente descrita en la era pre-antibiótica como extremadamente letal y debida habitualmente a una complicación tardía de una infección pleuropulmonar contigua ${ }^{1}$. Hoy en día es una patología inhabitual, en la que se observa un cambio en la frecuencia relativa de su etiología, lo que ha llevado a catalogar al Streptococcus pneumoniae, antes principal agente, una rareza en la actualidad ${ }^{1}$. Entre los mecanismos involucrados en la patogenia de la PP se encuentran: la extensión por contigüidad de infecciones del parénquima pulmonar, la diseminación directa por infecciones mediastínicas o la siembra por vía hematógena ${ }^{1,2}$.

En esta comunicación describimos el caso de un lactante de tres meses, previamente sano, quién consultó por fiebre y dificultad respiratoria de pocas horas de evolución, presentando una pericarditis neumocócica de foco primario desconocido, el cual evolucionó rápidamente hacia un shock cardiogénico con riesgo vital.

El objetivo de presentar este caso es destacar una complicación importante de la infección neumocócica, enfatizando en la necesidad de un rápido diagnóstico y tratamiento de la enfermedad neumocócica invasiva. Asimismo se revisa la literatura médica publicada al respecto para epidemiología, etiología, presentación clínica y opciones terapéuticas.

\section{Caso clínico}

Paciente de 3 meses de edad, sexo masculino, sano. Consultó por palidez, rechazo alimentario y dificultad respiratoria de seis horas de evolución. Al examen se encontraba pálido, quejumbroso, con frecuencia cardíaca en 142/ min y T $37^{\circ} \mathrm{C}$. Radiografía de tórax: sin foco de condensación, Índice Cardiotorácico (ICT) 60\% (figura 1). En exámenes de sangre destacó leucocitosis de 24 900/. $\mathrm{mm}^{3}$ y PCR en $183 \mathrm{mg} / 1$ $(\mathrm{VN}<10 \mathrm{mg} / \mathrm{l})$. Previo hemocultivo se inició tratamiento con vancomicina y ceftriaxona. Evolucionó en malas condiciones, con hepatomegalia progresiva, tonos cardiacos apagados. Se realizó intubación endotraqueal, conexión a ventilación mecánica, desarrollando shock resistente a catecolaminas. Ecocardiograma mostró derrame pericárdico extenso (11-14 mm hacia los lados y 12-18 $\mathrm{mm}$ por ápex) con imágenes hiperecogénicas en su interior. Se realizó pericardiocentesis de urgencia bajo ecografía que evidenció líquido citrino, coagulado. TAC de tórax y abdominal descartó foco mediastínico y abdominal. Se confirmó presencia de bazo. En quirófano se realizó ventana pericárdica, drenando $50 \mathrm{ml}$ de líquido opalescente y se dejó drenaje. Evolucionó grave, precarga optimizada hasta $150 \mathrm{ml} / \mathrm{kg}$ en el primer día, uso de drogas vasoactivas y esteroides en dosis de suplementación. Presentó derrame pleural bilateral, ascitis y síndrome compartimental abdominal con presión intrabdominal (PIA) hasta 16-18 mmhg. A las 24 horas se informó Hemocultivo $(+)$ para Streptococcus pneumoniae, la identificación de este microorganismo se realizó mediante métodos convencionales, resultando susceptible a los siguientes antimicrobianos, cefotaxima C.M.I. 0,016 $\mu \mathrm{g} /$ $\mathrm{ml}$; penicilina G C.M.I. 0,032 $\mu \mathrm{g} / \mathrm{ml}$ ajustando esquema a $200000 \mathrm{UI} / \mathrm{kg} / \mathrm{d}$. Confirmación en Instituto Salud Pública: Streptococcus pneumoniae no serotipificable.

Luego de drenaje quirúrgico y control de 


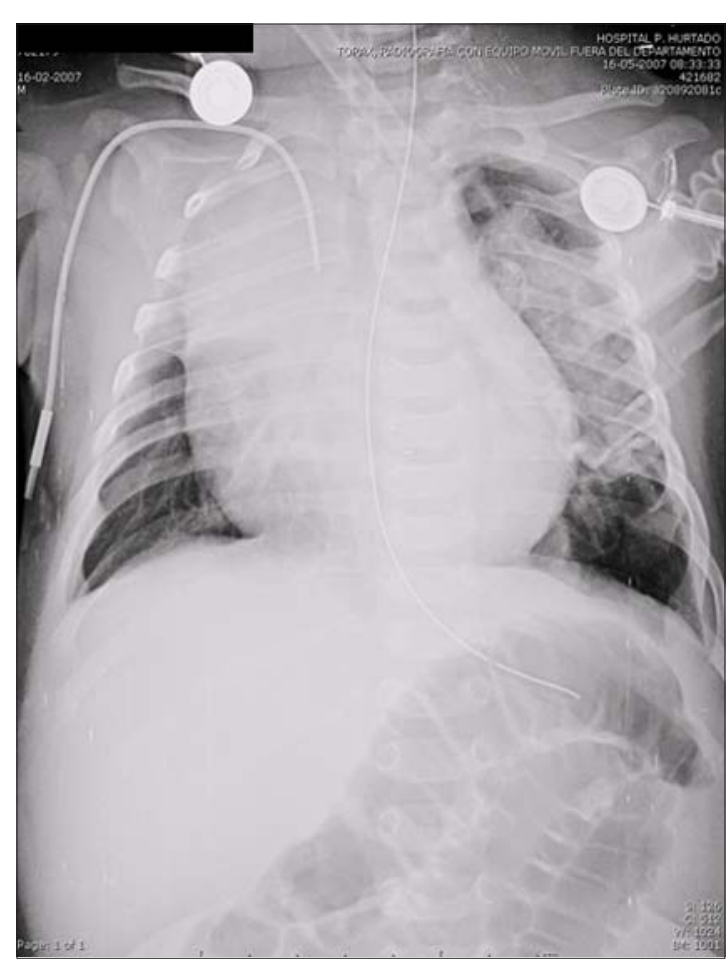

Figura 1.

infección presentó evolución favorable, es extubado al quinto día, previo control ecocardiográfico, se retiraron drenajes al octavo día. Cultivo de líquido pericárdico y ascítico fueron negativos. Completó tres semanas de tratamiento antibiótico en forma ambulatoria, y recibió vacuna antineumocóccica. El estudio inmunológico efectuado fue normal. Seguimiento a los doce meses, con paciente sano, asintomático desde el punto de vista cardíaco.

\section{Discusión}

La pericarditis aguda en población adulta es atribuida en casi un $90 \%$ a una causa viral o desconocida. En el resto de los casos esta puede ocurrir en asociación con otros agentes infecciosos (bacterias, mycobacterias y hongos), tumores (linfomas, rabdiomiosarcomas), causas metabólicas (uremia), desórdenes del tejido conectivo o enfermedades autoinmunes, en relación a infarto miocárdico, síndrome post pericardiotomía o en relación al uso de drogas (hidralazina, antiarrítmicos, anticonvulsivantes y anticoagulantes).

En niños, la pericarditis aguda es menos frecuente que en adultos, siendo la causa infecciosa la principal, atribuyéndose principalmente a infecciones virales por adenovirus, influenza o coxsackie (grupo B). Por otro lado, la PP es infrecuente y el aislamiento del agente bacteriano es en ocasiones difícil. Diversas bacterias han sido reportadas como causantes de PP, incluyendo Staphylococcus aureus, Neisseria meningitidis, Streptococcus viridans, Haemophilus influenzae, Streptococcus pyogenes $\mathrm{y}$ menos frecuentemente bacilos gram negativos entéricos y anaerobios ${ }^{2}$.

Antes de la incorporación de los antibióticos, la etiología neumocócica era la más prevalente, sin embargo, en la actualidad el Staphylococcus aureus es el agente más frecuente ${ }^{2}$. La presencia concomitante de otras infecciones puede ser a veces muy útil para la sospecha etiológica de la PP; Haemophilus influenzae y neumococo en relación a infección pleuropulmonar (neumonía o empiema) y Staphylococcus aureus, para osteomielitis y artritis séptica. En la gran mayoría de los casos la PP neumocócica es el resultado ya sea de la extensión directa o de bacteremia a partir de una infección intratoráxica tales como mediastinitis, neumonía, empiema, absceso miocárdico o endocarditis. La infección primaria pericárdica, es decir, sin foco previo, es aún más rara $\mathrm{y}$ podría ser producida por mecanismos patogénicos propios del microorganismo o bien causada por una condición de inmunodeficiencia del huésped.

En una reciente comunicación de Cakir y cols, para un período de diez años, se reportaron 18 casos, con una mediana de edad de 4 años, en donde la forma de presentación más frecuente fue fiebre y taponamiento cardíaco. Staphylococcus aureus fue el agente etiológico más frecuente y el factor predisponente más habitual fue la infección respiratoria. El drenaje subxifoideo y pericardiotomía fueron las opciones terapéuticas más usualmente empleadas. En esta casuística la mortalidad observada fue de un $5,5 \%{ }^{3}$.

En otra importante serie pediátrica, en don- 
de se excluyeron a los pacientes cardiópatas, la PP representó el 16\% de los casos de pericarditis, en un $42 \%$ de ellas no se encontró un foco infeccioso extrapericárdico. El agente más frecuente fue el Staphylococcus aureus (31\%), el Streptococcus pneumoniae fue aislado en el 16\%. Finalmente, el 21\% de ellos desarrolló pericarditis constrictiva requiriendo pericardiotomía ${ }^{4}$.

El caso aquí comunicado, demuestra que cepas susceptibles de Streptococcus pneumoniae pueden causar excepcionalmente PP por siembra hematógena. Sin embargo, un incremento en la resistencia en este agente patógeno adquirido en la comunidad, podría llevar a futuro a un incremento de las complicaciones supurativas.

\section{Fisiopatología}

La presencia de líquido en el espacio pericárdico superior a lo normal ( $1 \mathrm{ml} / \mathrm{kg}$ o hasta 15 ml de líquido) acompaña casi constantemente a la pericarditis clínica, de forma que la ausencia de derrame a ciertas edades, hace difícil sustentar el diagnóstico de pericarditis.

La patogenia de síntomas y signos está determinada por el aumento de la presión intrapericárdica, el cual depende no sólo de la cantidad absoluta de líquido sino también de la rapidez de su instalación y de las características del saco pericárdico. La acumulación rápida es peor tolerada que la acumulación lenta, la que permite un gran acumulo de líquido sin producir síntomas, siendo a veces en esta ocasión la primera manifestación los síntomas secundarios a compresión de estructuras vecinas (disfagia, tos, disnea) ${ }^{5}$.

El taponamiento cardíaco es un síndrome clínico-hemodinámico resultante del inadecuado llenado diastólico, presenta un continuum en cuanto a sucesión de gravedad, que puede ir desde ligeros aumentos de la presión intrapericárdica sin repercusión clínica reconocible, hasta un cuadro grave de hipodébito y muerte ${ }^{6}$.

\section{Diagnóstico}

\section{Manifestaciones clínicas}

En diagnóstico clínico es difícil, ya que en los niños prevalecen los síntomas inespecíficos, así el frecuente dolor precordial, síntoma predominante en adultos, puede pasar inadvertido en niños pequeños o manifestarse por disconfort e intranquilidad.

Dentro de los signos al examen físico, debe buscarse la caída de la presión de pulso, presencia de pulso paradójico, tonos cardíacos apagados, frotes pericárdicos, ingurgitación yugular, hepatomegalia y ascitis. Dentro de los exámenes de laboratorio el aumento de la silueta cardíaca puede ser un elemento clave.

\section{Radiografia de tórax}

A pesar de que cualquier aumento de la silueta cardíaca con campos pulmonares indemnes sugiere la presencia de efusión pericárdica, la radiografía de tórax puede no ser útil en un comienzo, ya que son necesarios al menos $200 \mathrm{ml}$ de fluido acumulados, antes de apreciar un aumento de la silueta cardíaca. En la proyección lateral, la presencia de línea grasa pericárdica es infrecuente, pero altamente sugerente de efusión pericardica masiva?

\section{Electrocardiograma}

La elevación del segmento ST y la depresión del voltaje son raras de observar en la $\mathrm{PP}^{8}$. En caso de derrame extenso, puede detectarse bajo voltaje generalizado y en ocasiones alternancia eléctrica (variaciones cíclicas de la amplitud de QRS).

\section{Ecocardiografía}

La ecocardiografía es la técnica de elección para el diagnóstico, cuantificación y seguimiento del derrame. Se exige que la separación de ambas hojas pericárdicas se observe durante todo el ciclo cardíaco. Pequeñas cantidades de líquido tienden a acumularse inicialmente entre la pared del ventrículo izquierdo y el pericardio posterior. Al aumentar la cantidad de líquido este puede envolver por completo al corazón y ser visible una separación anterior, entre el ventrículo derecho y el pericardio anterior. A veces, los derrames son encapsulados (frecuentes en derrames post cardiocirugía) y circunscritos a determinadas regiones.

Para el diagnóstico de taponamiento cardíaco, los signos ecocardiográficos más sensibles parecen ser el colapso telediastólico de la aurí- 
cula derecha y el colapso diastólico ventricular derecho ${ }^{5,6,9}$.

\section{Enzimas cardiacas}

La elevación en los niveles plasmáticos de troponina es ocasionado por la inflamación epicárdica más que por la necrosis de los miocitos. Una elevada concentración de troponina no se correlaciona con un pronóstico adverso, si no que una prolongación en el tiempo (mayor de dos semanas), sugiriendo la coexistencia de miocarditis, la cual ensombrece el pronóstico ${ }^{10}$.

\section{Tratamiento}

El diagnóstico precoz en combinación con una adecuada terapia que incluya antibioterapia y drenaje quirúrgico ha logrado un descenso de la mortalidad a cifras cercanas al $10-20 \% \%^{2,3}$.

En espera del cultivo definitivo la opción antibiótica debe de incluir un agente antiestafilocóccico. Vancomicina debe de considerarse en áreas de elevada tasa de resistencia estafilocóccica. No existe consenso sobre la duración óptima de la antibioterapia, siendo esta empírica, sin embargo, la mayoría de los reportes emplean entre 2 a 4 semanas de tratamiento endovenoso.

Debemos tener presente que la pericardiocentesis es vital como un procedimiento de salvataje, pero un procedimiento definitivo es necesario para evitar complicaciones (pericarditis constrictiva). Sin embargo, aún no hay consenso en la forma más segura y eficaz de lograrlo, existiendo todavía debate sobre lo necesario de la pericardiectomia y cual técnica de drenaje pericárdico es la más adecuada.

El uso de streptokinasa intrapericárdica fue inicialmente reportado por Juneja y cols ${ }^{11}$. En una reciente comunicación de Ekim y Demirbag, esta se empleó en nueve pacientes, siendo una medida eficaz para disolver las capas de fibrina y remover loculaciones, no observándose efectos secundarios de importancia como hemorragia sistémica, hipotensión arterial, arritmias o reacciones alérgicas ${ }^{12}$. Complicaciones reportadas infrecuentes con el uso de streptokinasa son el desarrollo de aneurismas por el debilitamiento del colágeno ${ }^{13}$. Las dosis sugeridas varían entre 10000 a $18000 \mathrm{U} / \mathrm{kg}$ disuelta en 50 $\mathrm{ml}$ de solución isotónica a temperatura corpo- ral, cada 12 horas por entre 3 a 8 dias, luego de lo cual se recomienda posicionar al paciente. Sin embargo, el agente fibrinolítico a emplear, la dosis y duración del tratamiento es materia aún de controversia.

Así entonces, la fibrinolisis intrapericárdica es una opción que puede en ocasiones servir para minimizar el riesgo de constricción pericárdica. No obstante la mayoría de las comunicaciones reportadas sobre su uso son casuísticas pequeñas ${ }^{14-16}$. Se debe de contar con trabajos a mayor escala para sugerirlo como una real opción terapéutica.

\section{Pronóstico y secuela}

Factores de mal pronóstico identificados, además de la instauración de un tratamiento tardío, son la presencia de taponamiento al momento del diagnóstico, la infección por Staphylococcus aureus o bacilos gram negativos y comorbilidades como la desnutrición.

La mayoría de los pacientes evoluciona sin secuelas, sin embargo, la más grave de observar es la pericarditis constrictiva. Otra secuela grave descrita es el desarrollo de aneurisma micótico aórtico ${ }^{17}$.

\section{Comentario}

Aunque esta enfermedad en la actualidad presenta una escasa incidencia, puede presentarse en una forma extremadamente agresiva, debido a cambios en los factores de riesgo y la resistencia antibiótica creciente, llevando en ocasiones al paciente a un compromiso vital, por lo que el médico debe de mantener un alto índice de sospecha.

\section{Referencias}

1.- Hästbacka J, Kolho E, Pettilä V: Purulent pneumococcal pericarditis: a rarity in the antibiotic era. $J$ Crit Care 2002; 17: 251-4.

2.- Roodpeyma S, Sadeghian N: Acute pericarditis in childhood: a 10-year experience. Pediatric Cardiol 2000; 21: 363-7.

3.- Cakir O, Gurkan F, Balci AE, Eren N, Dikici B: Purulent pericarditis in childhood: ten years of experience. J Pediatr Surg 2002; 37: 1404-8.

4.- Thébaud B, Sidi D, Kachaner J: Purulent pericarditis 
in children: a 15 year- experience. Arch Pediatr 1996; 3: 1084-90.

5.- Sagristá Sauleda J, Almenar Bonet L, Angel Ferrer J, et al: Guías de práctica clínica de la Sociedad Española de Cardiología en patología pericárdica. Rev Esp Cardiol 2000; 53: 394-412.

6.- Sagristá Sauleda J: Decisión clínica basada en técnicas de imagen de diagnóstico cardíaco. Diagnóstico y manejo terapéutico del paciente con taponamiento cardíaco o pericarditis constrictiva. Rev Esp Cardiol 2003; 56: 195-205.

7.- De Benedetti E, Didier D: Images in clinical medicine. Constrictive pericarditis. N Engl J Med 2000; 343: 107.

8.- Majid AA, Omar A: Diagnosis and management of purulent pericarditis. Experience with pericardiectomy. J Thorac Cardiovasc Surg 1991; 102: 413-7.

9.- Losekoot TG, Becker AE: Pericarditis. En Pediatric Cardiology. Robert H. Anderson et al. Ed. Churchill Livingstone. 1987; 1171-77.

10.- Imazio M, Demichelis B, Cecchi E, et al: Cardiac troponin I in acute pericarditis. J Ame Coll of Cardiol 2003; 42: 2144-8.
11.- Juneja R, Kothari SS, Saxena A, Sharma R, Joshi A: Intrapericardial streptokinase in purulent pericarditis. Arch Dis Child 1999; 80: 275-7.

12.- Ekim H, Demirbag R: Intrapericardial streptokinase for purulent pericarditis. Surg Today 2004; 34: 56972.

13.- Peuhkurinen KJ, Risteli L, Melkko JT, Linnaluoto M, Jounela A, Risteli J: Thrombolytic therapy with streptokinase stimulates collagen breakdown. Circulation 1991; 83: 1969-75.

14.- Defouilloy $C$, Meyer $G$, Slama $M$, et al. Intrapericardial fibrinolysis: a useful treatment in the management of purulent pericarditis. Intensive Care Med 1997; 23: 117-8.

15.- Winkler WB, Karnik R, Slany J: Treatment of exudative fibrinous pericarditis with intrapericardial urokinase. Lancet 1994; 344:1541-2.

16.- Mann-Segal DD. The use of fibrinolytics in purulent pericarditis. Intensive Care Med 1999; 25: 338-9.

17.- Albala MM, Meissner GF, Wachtel TJ, Fagan M: Case report: Rhode Island Hospital. R I Med J 1985; 68: 35-4. 